\title{
Designing and Detecting Trapdoors for Discrete Log Cryptosystems
}

\author{
Daniel M. Gordon* \\ Department of Computer Science, University of Georgia, Athens, GA 30602
}

\begin{abstract}
Using a number field sieve, discrete logarithms modulo primes of special forms can be found faster than standard primes. This has raised concerns about trapdoors in discrete log cryptosystems, such as the Digital Siguature Standard. This paper discusses the practical impact of these trapdoors, and how to avoid rhem.
\end{abstract}

\section{Introduction}

The National Institute of Standards and Technology (NIST) recently announced a proposal for a federal digital signature standard, DSS [21]. This proposal gives an algorithm for electronically signing documents, 10 guarantee the integrity of the message and the identity of the sender. The Digital Signature Algorithm (DSA) given in the proposal is based on the difficuly of solving discrete logarithms modulo large primes. It has atready excited a great deal of discussion regarding its efficiency and security.

In the DSA, the public key consists of a prime of 512 bits, a prime $q$ dividing $p-1$ of 160 bits, and a number $g$ which is a $((p-1) / q)$ th power mod $p$. The private key is a number $x$, and $y=g^{5}$ is also made public. Then to sign a message $m$, the sender calculates

$$
r \equiv\left(g^{k} \bmod p\right) \bmod q
$$

and

$$
s \equiv\left(k^{-1}\left(h(m)+x^{2}\right) \bmod q .\right.
$$

Here $H$ is any onf-say hash function, $m$ is the message, and $k$ is a random number less than q. To authenticate a message a recipient computes:

$$
\begin{gathered}
w=s^{-1} \bmod q . \\
u_{1}=(H(m) w) \bmod I, \\
u_{2}=(\operatorname{ro}) \bmod q .
\end{gathered}
$$

* Current address: Center for Communications Research, 4320 Westerra Court, San Diego, CA 92121

E.F. Brickell (Ed.): Advances in Cryptology - CRYPTO '92, LNCS 740, pp. 66-75, 1993.

(c) Springer-Verlag Berlin Heidelberg 1993 


$$
v=\left(g^{u_{1}} y^{2 w_{2}} \bmod p\right) \bmod q .
$$

The signature is correct if $v=r$.

The only known way to break this system is to find $x$ from $g$ and $y$ (i.e. find the discrete logarithm $\log _{g} y$ mod $p$ ). Several other schemes (e.g. [3], [6], [18]) also depend on the difficulty of discrete logarithms. Subexponential algorithms are known for finding discrete logarithms modulo large primes, but the largest prime for which the problem has been solved is 224 bits in length, by LaMacchia and Odlyzko [9], using the Gaussian integer method of Coppersmith, Odlyzko and Schroppel [5].

In [7], an algorithm is given for finding discrete logarithms using a number field sieve, which is asymptotically faster than other known methods. The general number field sieve is impractical, but a variant of the algorithm for primes of special forms is practical. The idea of using the number field sieve to make trapdoor primes is mentioned in [1], page 50 .

In Sect. 2. we give a brief description of how the special number field sieve for discrete logarthms works. Estimates for the time to break the DSS with regular versus various trapdoor primes are given in Sect. 3. The rest of the paper deals with how to detect trapdoors, how to construct trapdoors to avoid detection, and how one or nore people can choose primes for which the probability of a trapdoor existing is negligible.

\section{The Number Field Sieve}

Here we give a short presentation of the special number field sieve. For a more complete description of the algorithm, and the heuristic assumptions involved, see $[7]$.

Let $p$ be a prime and $f$ be an irreducible monic polynomial of degree $k$ with reasonably suall coefficients. such that for some integers $X$ and $Y$ near $p^{1 / k}$ we have $\gamma^{k} f(X / Y) \equiv 0 \quad(\bmod p)$. Let $\alpha \in \mathbb{C}$ denote a root of $f$, and $K=\mathbb{Q}(\alpha)$. For constructing traptoor primes, it is conveniont to pick $f$ so that $\hat{O}_{K}=\mathbb{Z}[\alpha]$ is a unique factorization domain.

We may define a homomorphism $\varphi$ from $\mathbb{Z}[\alpha]$ to $\mathbb{Z} / p \mathbb{Z}$ by sending $\alpha$ to $X / Y \bmod p$, so that for any integers $c$ and $d$,

$$
c Y+d Y=Y(c+d X / Y) \equiv Y p(c+d \alpha)(\bmod p) \text {. }
$$

The factor base $\mathcal{B}$ will consist of rational primes less than a bound $B\left(\mathcal{B}_{\mathbb{Q}}\right)$, first-degree primes in $\mathcal{O}_{K}$ with norm less than $B\left(\mathcal{B}_{K}\right)$, a fundamental set of units in $\mathcal{O}_{K}$, and $Y$. Calculating the primes and units for the field is not difficult when $f$ is, say $x^{5}-2$ (see [14]), but will be more difficult for polynomials with larger coefficients. He will discuss this problem in the next section.

Call a rational or algebraic integer smooth if its prime factors are all in the factor base. We will need to find many pairs of coprime integers $c, d$ such that $c Y+d X$ and $c+d a$ are both smooth. This can be accomplished efficiently by sieving $c Y+d X$ and the norm

$$
|N(c+d \alpha)|=\left|(-d)^{k} f(-c / d)\right|
$$


for fixed $a$ and large range of $d$. The smoothness of $c+d \alpha$ and $V(c+d a)$ are related by the following (see [7], Proposition 2):

Theorem 1. If $c$ and $d$ are relatwely prime and $r^{\prime} \| N(c+d a)$ for a prime $r$, then $\left(r, \alpha-c_{r}\right)^{d} \|(c+d \alpha)$ in $\mathcal{O}_{K}$, for $c_{r} \equiv-c / d \quad(\bmod r)$.

We will choose $g$, the base for the discrete logarithm to be smooth and a primitive root modulo $p$. Note that this cannot be the same as the base $g$ for the DSA, since that $g$ is a $(p-1) / q$ th power. Thus, the first step in breaking the DSS would be to find the log of its base.

The precomputation step involves sieving through small $c$ and $d$. looking for pairs with $c Y+d X$ and $N(c+d \alpha)$ both smooth. Each hit gives us an equation involving logarithms of the factor base. Suppose that we find a $c$ and $d$ for which both are smooth, say

$$
c\rangle+d x=\prod_{s \in B_{d 2}} s^{w \cdot(c \cdot d)}
$$

and

$$
\mid N\left(c+d(x) \mid=\prod_{s \in b_{\mathbb{Q}}} s^{u s(c, d)}\right.
$$

for $v_{s}, w_{s} \in \mathbb{Z}_{\geq 0}$. Then

$$
(r+d x)=\prod_{s \in b_{n}} s^{k s(a d)}
$$

by Theorem 1. Since $\mathcal{O}_{K}$ is a [FD, this equation involving ideals can be replaced with one involving algebratic intigers. by replacing each s in the above equation by a generator for the iden. Then $c+d a$ divided by the generators is a unit, which can be explicilly computed in terms of a fundamental set of units, using Theorem 5 of $[7]$.

From this, we obtain:

$$
(c Y+d X) \varphi(c+d \alpha)^{-1} \equiv \prod_{s \in \mathcal{B}} s^{u_{s}(c, d)} \equiv 1 \quad(\bmod p)
$$

which gives us an equation for the logs of the factor base:

$$
\sum_{x \in L^{i}} u_{s}(c, d) \log _{g} s \equiv 0 \quad(\bmod p-1)
$$

Once we have more than $|B|$ hits, we solve the resulting matrix equation over $\mathbb{Z} /(p-1) \mathbb{Z}$ using structured Gaussian elimination to reduce the size of the matrix, and then solving a smaller, dense matrix using the conjugate gradient method or Wiedemann's algorithm (see [10]). This completes the precomputation.

To find an individual logarithm. we reduce the problem to finding the logs of medium-sized primes. Choose random values of $s$ and attempt to factor $g^{3} y$ $(\bmod p)$ using the elliptic curve method (ECM) until one is found for which

$$
y^{*} y \equiv q_{1} q_{2} \cdots q_{r} \quad(\bmod y)
$$


with each $q_{i}$ is less than a bound $Q$. (This can be improved as in [9], by finding $z_{1}, z_{2}=O(\sqrt{p})$ such that $g^{3} y \equiv z_{1} / z_{2} \quad(\bmod p)$, and testing whether $z_{1}$ and $z_{2}$ are both $Q$-smooth.)

For each $q_{i}$, we will sieve $c$ and $d$ for which $q_{i} \mid(c \xi+d X)$, say fixing $d$ and taking $c=c_{0}+e q_{i}$, to find one value for which $(c Y+d X) / q_{i}$ and $N(c+d \alpha)$ are both smooth. Once this happens we are done, since from the precomputation we know the logs of the whole factor base.

The choices for the size of the factor base and $q_{i}$ 's depend on how time is to be divided between the two stages. Enlarging the factor base reduces the time needed to find individual logarithms, but at the cost of increasing the precomputation time. Lot

$$
L_{n}[v ; c]=\exp \left\{(c+o(1))(\log n)^{u}(\log \log n)^{1-v}\right\}
$$

for $n-\infty$. Assuming some reasonable heuristics (see [i]), the optimal choice of paranteters is

$$
\begin{gathered}
k=\left[10^{1 / 5}\left(\frac{\log p}{\log \log p}\right)^{1 / 5}\right] . \\
B=L_{y}\left[2 / 5:(1 / 125)^{1 / 5}\right] .
\end{gathered}
$$

a.nd

$$
\left.Q=L_{0}, 3 / 5 ;(1 / 100)^{i / 5}\right]
$$

which results in both the precomputation and individual logarithms taking expected rime

$$
L_{\mathrm{p}}\left[2 / 5:\left(\frac{128}{125}\right)^{1 / 5}\right] \approx L_{p}[2 / 5: 1.00475] .
$$

If llany instances are to be done for one $p$. more time could be spent on the precomputation by taking a larger factor base. for $\mu \geq(128 / 125)^{1 / 5}$, if we spend $L_{n}[2 / 5 ; \mu]$ time on the pecomputation, each logarithm can be found in time

$$
L_{p}\left[2 / 5:\left(\frac{128}{125 \mu^{2}}\right)^{1 / 3}\right]
$$

The Gaussian integer method is a special number field sieve with $k=2$ and $K$ a complex quadratic field. For any $c \geq 1$, the Gaussian integer method can find logarithms in time $L_{p}[1 / 2 ; 1 /(2 c)]$ if $L_{p}[1 / 2 ; c]$ is spent on the precomputation. Even for fairly small primes with good polynomials, the special number field sieve is faster than the Gaussian integer method.

For primes which cannot he represented by good polynomials, a similar procedure called the general number field sieve can be done. The difference is that the polynomial $f$ will have large coefficients, so operations in the resulting field will be impractical. To avoid them, the equations must be solved over the rationats instead of moduto $p-1$, to eliminate ideals and units.

The better asymptotic time for the general number field sieve comes from using different fields for finding individual logarithms. Instead of sieving through 
$c$ and $d$ such that $q_{i} \mid(c Y+d X)$, we search through polynomials for which $q_{i} \mid \varphi(\alpha)$. This allows us to take $Q$ as big as $X$ and $Y$, which asymptotically speeds up the algorithm. The time for the general number field sieve is

$$
L_{p}\left[1 / 3 ; 3^{2 / 3}\right] \approx L_{p}[1 / 3 ; 2.08]
$$

Oliver Schirokauer [17] lass developed a method to avoid solving equations over the rationals, so that the time can be improved to $L_{p}[1 / 3,1.902]$. The larger constant and o(1) terms make the general number field sieve impractical for numbers we are incerested in.

\section{Complexity Estimates}

There are four parts of the algorithm which dominate the timing estimates. For the precomputation, there is the sieve to gather equations, and then the linear algebra modulo $p-1$ to solve the equations. For finding individual logarithms, the medium-sized primes are found by repeated trials of the ECM, and then another sieve must he dome for each q

How much time is devoted to each part depends on the choice of parameters: the degree of of he polymonial $f$, the polyomial chosen (and the resulting field). the size of the factor bast. and he size of a medium-sized prime.

For $k=2$, we can take $f=x^{2}+r$ for $r$ a small positive integer for which $-r$ is a quadratic residur modulo $p$. lhen the resulting field is just a complex quadratic field $Q(\sqrt{-r})$, and we hatve the Gaussian imeger method. This can be applied to any prime, but is impractical for 512-bit prines. Breaking the DSS using the Gaussian integer method using $B=50,000,000$ would require sieving $10^{20}$ numbers. Even if this could be accomplished, the resulting natrix would have over 5, 000, 000 columns, and the linear algebra problem would be a major hurdle.

The numbers in Table 1 show the difficulty of finding discrete logarithms for 512-bit primes using the special number field sieve with polynomials of degree 2-5 with small coefficionts. They assume that the large prime variation described in [14] is being used. They are intended as rough estimates only, but serve to give an idea of the tims resuired. For comparison. the factorization of $F_{9}$ required sieving about $10^{1.1}$ numbers. and solving a matrix with 199.203 columns modulo 2 [14]. For larger $k, X$ and $Y$ are smaller, so a smaller factor base can be used, speeding the precomputation. But then for individual logarithms $N(c+d \alpha) \approx Q^{k}$ is larger, so we need to take $Q$ smaller and do more ECM trials.

Table 1 indicates that the ideal polynomial for a trapdoor would have degree four. Its coefficients should be small, to keep down the size of $N(c+d \alpha)$. The field generated by a root of the polynomial should have small discriminant and regulator, class mumber one and index one, so that field operations can be done efficiently. If the polynomial has four comples roots. then the unit group will have rank one.

For example, the polynomital $x+x+1$ satisfies all the above conditions. The problem is that the polynomial could only be used with primes $p$ for which there 
Table 1. Statistics for 512 -bit primes with good polynomials.

\begin{tabular}{|c|c|c|c|c|}
\hline $\mathrm{k}$ & 2 & 3 & 4 & 5 \\
\hline$B$ & $5 \times 10^{7}$ & $5 \times 10^{6}$ & $3 \times 10^{6}$ & $2 \times 10^{6}$ \\
sieve range & $10^{20}$ & $2 \times 10^{16}$ & $2 \times 10^{14}$ & $10^{14}$ \\
matrix size & $5,600,000$ & 650,000 & 400,000 & 280,000 \\
\hline$Q$ & $10^{20}$ & $10^{19}$ & $10^{15}$ & $10^{13}$ \\
\# ECM trials & 29,000 & 78,000 & $2 \times 10^{7}$ & $2 \times 10^{9}$ \\
second sieve & $2.5 \times 10^{14}$ & $2.4 \times 10^{15}$ & $1.5 \times 10^{14}$ & $3 \times 10^{14}$ \\
\hline
\end{tabular}

exist $X, Y \approx p^{1 / 4}$ such that $X^{4}+X Y^{3}+Y^{4} \equiv 0(\bmod p)$. This is a thin set of primes, which can easily be detected (see the next section).

For polynomials with larger coefficients, the special number field sieve is more complicated. The sirtug nage takes shighty longer, since the norms being tested for smoothmess are larger. For polynomials with coefficients of say, up to 100 in absolute value, the sieving range musl be increased by roughly a factor of ten.

Another difficulty is dealing with a field of larger discriminant. The problem is finding generators for the unit group and prine ideals in the factor base. In [13], these are found by searching through algebraic integers of the form $\sum_{t=0}^{4} h_{i} \alpha^{i}$, for $\alpha$ a generator of $h$ and small values of $h_{2}$. For fields generated by polynomials with larger coefficients, this will be impractical

There have been several papers on efficient algorithms to fund units and algebraic integers of given noms in gemeral munter fields, (see [4], [16]). The computations are involved. but they only need to be done once for a given $f$.

The matrix equation resultugg from the sieving may be solved using intelligent

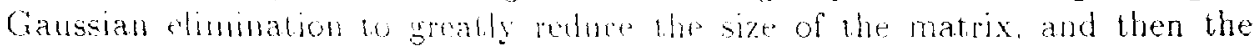
conjugate gradient algorithm to solve the reduced equation. In [10] these methods were used to solve matrices with up to 96.321 columns.

\section{Trapdoor Primes and Polynomials}

From Table 1, we see that some 512-bit prines nay not be sale, but general ones (at least for the moment) are. We want to ensure that for a given prime $p$ there is a no polyomial f which can be used for the special number field sieve. Currenty, the only wat to check for this is to check one polyomial at a time.

Let $p$ be a 512-bit prime and $f$ be a polynomial of degree $k \geq 3$. We will say that $I$ and $\gamma$ are a trapdoor for $p$ and $f$ if they are both less than (say) $1,000 p^{1 / k}$ in absolute value. arid $\gamma^{6} f(K / \gamma) \equiv 0(\bmod p)$.

Theorem 2. If a trapdoor K. Y exists for $p$ and $f$. then for a rool $c_{p}$ of $f$ mod $p$, $(X, Y)$ is a short vector in the latice $\mathcal{L}=\left\langle(p, 0),\left(c_{p}, 1\right)\right\rangle$.

Proof. Let $c_{p}$ be the root of $f \bmod p$ congruent to $X / Y \bmod p$. The lattice $\mathcal{L}$ contains $(X, Y)$, since $\left.\left(c_{p}, 1\right)\right\}^{\circ}=\left(c_{p} Y, Y\right) \equiv(X, Y)(\bmod p)$. 
The shortest vector in $\mathcal{L}$ has length at most. $O(\sqrt{p})$, and for most choices of $p, f$ and $c_{p}$, the short vector will be $\theta(\sqrt{p})$. If such an $X$ and $Y$ do exist, $(X, Y)$ has length $<\sqrt{2} 1.000 p^{1 / k}$

Conversely, all vectors $\left(X^{\circ}, Y\right) \in \mathcal{L}$ satisfy $\left.\}^{*} f\left(\mathrm{~K}^{*} /\right\}^{\circ}\right) \equiv 0 \quad(\bmod p)$, so any such short vector is a trapdoor for $f$ and $p$.

This gives an efficient algorithm for testing whother a trapdoor exists for a given $f$ and $p$. One may find linear factors of $f \bmod p$ efficiently by eliminating square factors (dividing by the greatest common divisor of $f$ and $f^{\prime} \bmod p$ ), and then taking the gcd of $\left(x^{p}-x\right)$ and $f \bmod p$ (see [8]). Then $X$ and $Y$, if they exist, can be found using lattice reduction.

The main problem with this is that every polynomial $f$ needs to be considered separately, so a limited range of polynomials can be searched. On a Sparcstation 1, one fourth-dogree polynomial can be tested for a 512 -bit prime in about a minute. On a parallel machine many polynomials could be searched at once, and a fairly latge mage of polynomale could be tested. Nith this test, a trapdoor with a good polymomial could be fomm. This forces an adversary to choose a. polynomial from a set too latge to be exhanstively searched, say an $f$ of degree fourth with coefficients chosen rambnily between -100 and 100 .

Note that in the special cas $Y=1$, where $\mu=f(X)$, the polynomial can be found much faster. In this case $\left(p / a_{k}\right)^{1 / k}$ is close to $Y$, where $a_{k}$ is the leading coefficient of $f$. All polynomials with a given $a_{k}$ could be tested at once, very efficiently, so such a trapdoor would be much easier to discover.

Similar techniques can be used to construct a trapdoor prime. Suppose we wish to compute y and p lor the DSA steh that for a giten polynomial $f(x)=$

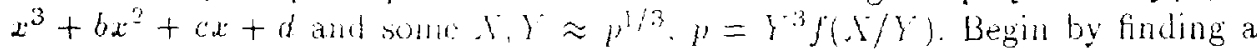
160-bit prime $q$, and choosing any $Y_{0} \approx 2^{10}$. Let $g(x)=Y_{0}^{3} f\left(x / Y_{0}\right)$. Then we may find an a mod y swh lhat $y(a) \equiv 1$ (mod y). by looking for linear factors of $g(x)-1$ (mod $y$ ). If none exists, then mother $\gamma_{0}$ may be tried.

For any $X \equiv$ (mod $y)$ and $\zeta \equiv Y$ (mod $q)$, we have $Y^{3} f(X / Y) \equiv 1$ $(\bmod q)$. Taking $Y=a+l q$ and $\xi=\zeta_{3}+l_{2 q}$. with $l$ and $l$ g chosen so that $Y^{3} f(X / Y) \approx 2^{512}$. we expect to soon find a pair for which $p=Y^{3} f(X / Y)$ is prime. This $p$ and $q$ could be used as a trapdoor for the DSS.

This is not an ideal trapdoor, since from Table 1, a degree four polynomial would work better. The problem with constructing a better trapdoor using the above method is that a is usually a 160 -bit number. which is bigger than $p^{1 / 4}$, so $X$ would be too harge. The revised DSS will allow primes $p$ up to 1024 bits [19]. For primes with 640 or mor bits. the above method can be used to make a trapdoor with a degres four polynomial. For primes with 800 or more bits, a degree five polymomial can be ment.

Another way lo genterate a traploor would be to choose a polynomial $f$, and try random values of $X$ and $Y$ until $p=\gamma^{k} f(x / Y)$ is prime and divisible by a l60-bit prime q. To find such a value one could sieve by small primes or use the ECM factoring method to find an . Y. Y pair for which $p-1$ is smooth except for one 160-bit prime factor. This has the drawback that $(p-1) / q$ would be smooth, which while it is not known to weaken the system, does seem undesirable. 


\section{Protocols for Choosing a Prime}

The ideal way to avoid worries about a trapdoor would be to come up with a way of generating primes for which one can guarantee that no such polynomial exist.s. An alternative is to use a random prime, which is almost certain to be safe. Call a prime $p$ unsafe if an $f$ exists with $Y^{k} f(X / Y) \equiv 0(\bmod p)$, where $k$ is between 3 and $10, X$ and $Y$ are less than $1,000 p^{1 / k}$, and the absolute values of the coefficients of $f$ are less than 500 . Then the fraction of 512-bit primes which are unsafe is at most

$$
\frac{1}{\pi\left(2^{512}\right)-\pi\left(2^{511}\right)} \sum_{k=3}^{10}\left(1,000 \cdot 2^{512 / k}\right)^{2} \cdot 1000^{k+1}<2^{-100} .
$$

Suppose wo prople wish to agree on a sale key for the DSS. They can choose a random seed for the random number generator, using a protocol due to Blum [2]. From this they can use the method of Appendix 2 of [21] to create a key which is as likely to be satir as any randon bey

On the other hand, a cembal authority might wast to announce a key for general use, so that everyone is convinced there is no trapdoor. To do this, the authority must have a pseudo-random number generator and algorithm for constructing keys so that

1. Any uscr can ierify that a key was generated using the approved method.

2. Keys produced by this method should be no more litely than random keys to contain trapdoors.

3. The choice of sied used for the random number generator should not allow the authority to crates a particular bey

With a fow modification, he matom mumber generator mentioned in Appendix 3 of [21] can be made to satisfy the abote eriteria. That method uses DES with a 64-bit seed, DES key and 64-bit date/time-stamp. To satisfy the above conditions. the DES key used should lie fixed as part of the algorithm, the seed should be made public with the DSS key, and the time-stamp format should be specified.

It could be argued that the 64-bit seed gives too much freedom, putting the third condition at risk. This can be remedied by restricting the choice of seeds, or eliminating the seed entirely and just using the time-stamp.

For an example of a "tmotable" key, consider:

$$
q=1147860701762054730346201299935827782113538756127
$$

and

$$
\begin{aligned}
p= & 715619176139780204927878779193336180873773390583792476383 \\
& 44062581902861059517171507927020818420231820214082169894 \\
& 373334078735314126297272778927524812627411
\end{aligned}
$$


These numbers were generated using the binary expansions of $\pi$ and $e$. The prime $q$ is the smallest prime greater than $\left[2^{158} \pi\right]$, and $(p-1) / q$ is the smallest number larger than $\left[2^{350} e\right]$ for which $p$ is prime. There is no reason to suspect this number of being any more likely than a random number to have a trapdoor, and tests of $p$ by many polynomials have not found any.

\section{Conclusion}

In this paper, we have tried to quantify the threat of trapdoors for discrete logarithm-based cryptosystens, in particular DSS. While trapdoors do give a definite advantage over standard keys, with a lew easy precautions in the choosing of $p$ and $q$ it is possible to prevent them, and they do not seem to pose a major problem for such systems.

In [15], Maurer and Yacobi present a public key distribution system, based on computing discrete logarithms nodulo a composite number $n$. The factorization of $n$ is a trapdoor which allows a trusted authority to compute secret keys. Untike the DSS, their system enties on the trapdoor, and they ask if a similar trapdoor can be made for primes. The special mumber field sieve does provide a trapdoor

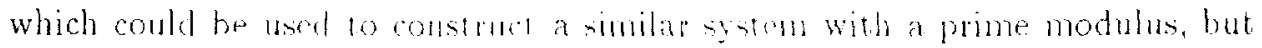
such a system would be impratical

\section{References}

1. T. Beth, M. Frisch and C..J. Simmons, eds. Pubic-key Cryptography, State of the Ant and Future Direction, LYCS \#578, Springer, 1992.

2. M. Blum. Coin flipping by telephone: A protocol for solving impossible problems, (Procedings of the 24th IFEF Computer Conference. 1982, pp. 133-137.

3. E.F. Brichell and K.S. Mcerutey du Faleractive ldentification Scheme Based on Discrete Logartitum ind Factoring, Jommal of Cryptology, to appear.

4. 1. Buclumann and A. Pethö. Conputation of independent units in number fields

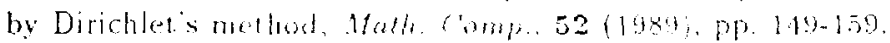

5. D. Coppersmith. A.M. Odlyzko and $R$. Schroeppel, Discrete logarithms in GF(P), Algorthmoca, 1 (1986). pp. 1-15.

6. W. Diffie and M.E. Hellman, New directions in cryptography, IEEE Trans. Info. Theory, $22(1976)$, pp. D44-6551.

7. D. Gordon, Discrete logarithms in $G F(p)$ using the un mber field sieve, SIAM Journal on Discrete Math.. to appear.

8. D.E. Kinuth, The Art of Computer Programming. Vol. 2, Seminumerical Algorithms, Second Edition, Addison-Wesley, Missachuselts, 1981.

9. B. LaMacchia and A.M. Onlyako. Computation of discrete logarithms in prime

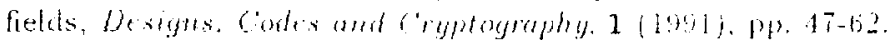

10. B. LaMacchia and A.M. Odlyzo, Solving large sparse linear systems over finite fields, Advances in Cryptology: Procedings of Crypto 90. (A. Menezes. S. Vanstone eds.), Lecture Votes in Computer bontor Springer-Verlag, New York. 1991.

11. H.W. Lenstra, Ir. Fachorng integers whth elliptic curves, Ann. of Math., 126 (1987), Pp. 649-673. 
12. A.K. Lenstra, U.W. Lenstra, Jr., and L. Lovása, Factoring polynomials with rational coefficients, Math. Ann. 261 (1982), pp. 515-534.

13. A.K. Lenstra, H.W. Lenstra, Jr., M.S. Manasse and J.M. Pollard, The number field sieve, Proc. 22nd ACM Symposium on Theory of Computing (1990) pp. 564-572.

14. A.K. Lenstra, H.W. Lenstra, Jr., M.S. Manasse and J.M. Pollard, The factorization of the ninth Fermat number, preprint, 1991.

15. U.M. Maurer and Y. Yacobi, A non-interactive public-key distribution system, Advances in Cryptology: Proceedings of Eurocrypt 91, (D.W. Davies, ed.), Lecture Notes in Computer Science, Springer-Verlag, New York, 1991, pp. 498-507.

16. M. Polıst and II. Zassenhaus, Algorithmic Algebraic Number Theory, Cambridge University Press, Cambridge, 1989.

17. O. Schirokaner, On pro-finite groups und on discrete logarithms, Ph. D. thesis, University of California, Berkeley, May 1992.

18. C.P. Schnorr, Efficient signatute generation by smart cards, Joumal of Cryplology, to appear.

19. M.E. Smid and D.K. Branstad, Response to comments on the NIST Proposed Digital Signature Standard, Advances in Cryplology: Proceedings of Crypto '92, to appear.

20. D.H. Wiedemarn. Solving sparse linear eguations over finite fields, IEEE Trans. Info. Theory. 32 (1986). pp. 54-632

21. Specifications for a digital signature standard, National Institute for Standards and Technology, Frderal Information Processing Standard Publication XX, draft, Aluguse ly!l. 